\title{
DIFFUSION TENSOR IMAGE PROCESSING USING BIQUATERNIONS
}

\author{
K. Krishna Nand ${ }^{\dagger} \quad$ Ghassan Hamarneh ${ }^{\star} \quad$ Rafeef Abugharbieh ${ }^{\dagger}$ \\ ${ }^{\dagger}$ Biomedical Signal and Image Computing Lab, The University of British Columbia \\ * Medical Image Analysis Lab, Simon Fraser University \\ \{kkrishna, rafeef $\} @$ ece.ubc.ca, hamarneh@sfu.ca
}

\begin{abstract}
We propose a biquaternion formalism to model diffusion tensor magnetic resonance imaging (DT-MRI) data. Unlike previous methods that use dimensionality reduction, we are able to process the full tensor in a holistic manner while respecting the underlying manifold of the data. Using this approach, we introduce the Fourier transform and convolution for DT-MRI for the first time, which can be applied directly on the full tensor. This opens up a wide range of applications for DTMRI image processing. Further, based on this formulation, we present a biquaternion gradient vector and edge detector for DT images. Preliminary results of applying the Fourier transform, convolution and edge detector on synthetic examples as well as real DT data show great promise in our approach for DT image processing.
\end{abstract}

Index Terms - diffusion tensor MRI, biquaternions

\section{INTRODUCTION}

DT-MRI is a powerful non-invasive imaging modality whose processing, analysis and visualization has become a strong focus in medical imaging research [1]. In this modality, the direction of the water diffusion is locally modeled by a Gaussian probability density function whose covariance matrix is a second order $3 \times 3$ symmetric positive definite matrix [2]. The manifold-valued nature of the data as well as its high dimensionality makes the computational analysis of DT-MRI complex. Very often, the data dimensionality is reduced to a single scalar derived from the tensors, e.g. fractional anisotropy (FA) [3]. Another common approach has been to ignore the restriction to the manifold of symmetric secondorder tensors and, instead, treat the data as a multi-valued image [4].

Quaternion representation has been proven useful for processing color images [5, 6]. Taking inspiration from that work, we propose a biquaternion (complexified quaternion) representation to model DT data. The basic idea is to represent the DT at each pixel by a biquaternion via the log-Euclidean transform [7]. The key innovation here is that we can now consider the DT as a whole in a holistic manner rather than as separate scalar channels or as a single scalar derived from the tensors. With this representation, we handle the coupling between the channels naturally while respecting the manifold of the symmetric second order tensors. Further, this approach for the first time, enables us to introduce tools from biquaternion theory such as Fourier transform and convolution for processing DT images. We also present a biquaternion gradient vector and edge detector for DT images that further demonstrate the applicability of this novel representation. Our approach opens new ways for carrying out DT image processing tasks including frequency filtering, convolution, compression, and interpolation. We present preliminary results of applying the Fourier transform on various synthetic and real DT images, which give the desired and expected results. We further demonstrate the application of our method to frequency filtering and compression. Finally, we show how our representation enables DT-MRI edge detection with favorable results over FA-derived edges. Our results demonstrate the potential of our approach for DT image processing.

\section{METHODS}

\subsection{Brief review of biquaternions}

We begin by reviewing biquaternions and some of their basic properties. Let us denote the set of complex numbers by $\mathbb{C}$ and the set of biquaternions by $\mathbb{H}_{c}$ (the set of Hamilton's quaternions are denoted by $\mathbb{H}$ ). Biquaternions are an 8-D algebra consisting of Hamiltonian quaternions with complex components and can be represented as $q=a+b i+c j+d k$, where $q \in \mathbb{H}_{c}, a, b, c, d \in \mathbb{C}$, and $i, j, k$ are the quaternion imaginary units. We have the following set of standard relations between $i, j, k: i^{2}=j^{2}=k^{2}=i j k=-1 ; i j=$ $-j i=k ; k i=-i k=j ; j k=-k j=i$. Hence, just like quaternions, biquaternion multiplication is non-commutative.

Let the elements of $\mathbb{C}$ be represented as $z=\Re(z)+$ $I \Im(z)$, where $I=\sqrt{-1}$ is the complex imaginary unit and $\Re(z), \Im(z) \in \mathbb{R}$. $i, j, k$ and $I$ are related as $i I=$ $I i ; j I=I j ; k I=I k$. This basically means that any complex co-efficient commutes with any quaternion imaginary unit. Thus biquaternions form an 8-D vector space with the basis $\{1, i, j, k, I, i I, j I, k I\}$ over $\mathbb{R}$. 
Any biquaternion can be seen as the sum of a scalar and a vector part as $q=S(q)+V(q)$, where $S(q)=a$ and $V(q)=b i+c j+d k$. A biquaternion with zero scalar part $(S(q)=0)$ is called a pure biquaternion. The total conjugate of a biquaternion is defined as $\bar{q}=a^{*}-b^{*} i-c^{*} j-d^{*} k$, where $a^{*}, b^{*}, c^{*}$, and $d^{*}$ are the complex conjugates of $a, b, c$, and $d$, respectively. Lastly, the norms of a biquaternion $q$ and a biquaternion vector $\boldsymbol{q} \in \mathbb{H}_{c}^{N}$ (a vector whose components are biquaternions) are given by

$$
|q|=\sqrt{S(\bar{q} q)} ; \quad\|\boldsymbol{q}\|=\sqrt{S\left(\sum_{n=1}^{N} \bar{q}_{n} q_{n}\right)},
$$

where $S(\cdot)$ is the scalar part and $q_{n}$ is the $n^{\text {th }}$ component of $\boldsymbol{q}$. It is to be noted that biquaternions do not form a normed division algebra and hence the norm of a biquaternion is not multiplicative. i.e. $|q r| \neq|q||r|$.

\subsection{Biquaternion representation for DT}

DTs do not form a vector space. In particular, the space of symmetric second order positive definite tensors $\operatorname{SPD}(3)$ is restricted to a convex half cone. Hence before we proceed to represent a DT by a biquaternion, we need to ensure that we respect the manifold of the DTs. For this, we employ the log-Euclidean (LE) transformation proposed in [7] to map the space of DTs into a vector space. Let $\boldsymbol{D}(\boldsymbol{x})$ denote a DT image indexed by $\boldsymbol{x}$ (i.e. $\boldsymbol{x}=\left[\begin{array}{ll}x_{1} & x_{2}\end{array}\right]$ in $2 \mathrm{D}$ and $\boldsymbol{x}=$ $\left[\begin{array}{lll}x_{1} & x_{2} & x_{3}\end{array}\right]$ in 3D). Then the LE transformation gives $\boldsymbol{L}(\boldsymbol{x})=$ $\operatorname{logm}(\boldsymbol{D}(\boldsymbol{x}))$, where $\boldsymbol{L}(\boldsymbol{x})$ is the LE tensor and $\operatorname{logm}(\cdot)$ is the matrix logarithm. $\boldsymbol{L}$ spans a vector space of symmetric matrices also known as the log-Euclidean space [7]. Let the components of $\boldsymbol{L}(\boldsymbol{x})$ be denoted by $\left(L_{0}, L_{1}, L_{2}, L_{3}, L_{4}, L_{5}\right)$. The $\log$-Euclidean tensor $\boldsymbol{L}(\boldsymbol{x})$ is represented by a pure biquaternion (whose scalar part is zero) in the following way:

$$
q(\boldsymbol{x})=\left(L_{0}+I L_{1}\right) i+\left(L_{2}+I L_{3}\right) j+\left(L_{4}+I L_{5}\right) k .
$$

The result of this representation is that we have now moved from the space of symmetric second order positive definite tensors to the vector space of pure biquaternions, while respecting the manifold of the tensors ${ }^{1}$.

\subsection{Fourier transform, convolution and gradient for DTs}

We introduce the Fourier transform for DTs based on the biquaternion Fourier transform [8]. Considering only a single biquaternion exponential kernel, we can define left and right Fourier transforms depending on the side by which the exponential kernel is multiplied. The left 2D DT Fourier transform

\footnotetext{
${ }^{1}$ Even though there are multiple ways to encode the components of $\boldsymbol{L}$ into $q$, any one choice will result in a specific representation that can be uniquely decoded back to the log-Euclidean space and the advantages/analyses that we present next still hold regardless of the choice made.
}

and its inverse are given by:

$$
\begin{aligned}
\hat{q}_{L}\left(f_{1}, f_{2}\right) & =\frac{1}{\sqrt{M N}} \sum_{x_{1}=0}^{M-1} \sum_{x_{2}=0}^{N-1} e^{-\mu 2 \pi\left(\frac{x_{1} f_{1}}{M}+\frac{x_{2} f_{2}}{N}\right)} q\left(x_{1}, x_{2}\right), \\
q\left(x_{1}, x_{2}\right) & =\frac{1}{\sqrt{M N}} \sum_{f_{1}=0}^{M-1} \sum_{f_{2}=0}^{N-1} e^{\mu 2 \pi\left(\frac{x_{1} f_{1}}{M}+\frac{x_{2} f_{2}}{N}\right)} \hat{q}_{L}\left(f_{1}, f_{2}\right),
\end{aligned}
$$

where $f_{1}, f_{2}$ are the frequency variables, $\mathrm{M}$ and $\mathrm{N}$ are the number of pixels along $x_{1}$ and $x_{2}$, and $\mu$ is a biquaternion root of -1 , also called the axis of the transform. The magnitude spectrum of the Fourier transform is computed by taking the norm of $\hat{q}_{L}$ using (1).

Convolution for 2D DT images is given by

$\left(q_{1} * q_{2}\right)\left(x_{1}, x_{2}\right)=\sum_{x_{m}=0}^{M-1} \sum_{x_{n}=0}^{N-1} q_{1}\left(x_{m}, x_{n}\right) q_{2}\left(x_{1}-x_{m}, x_{2}-x_{n}\right)$

where $q_{1}$ and $q_{2}$ are biquaternions.

Finally, the gradient vector and edge detector for 2D DT images using biquaternions is given by

$$
\begin{aligned}
& \boldsymbol{g}\left(x_{1}, x_{2}\right)=\left[\partial q\left(x_{1}, x_{2}\right) / \partial x_{1} \quad \partial q\left(x_{1}, x_{2}\right) / \partial x_{2}\right]^{T}, \\
& e\left(x_{1}, x_{2}\right)=\left\|\boldsymbol{g}\left(x_{1}, x_{2}\right)\right\|,
\end{aligned}
$$

where $\boldsymbol{g}$ is the biquaternion gradient vector and $e$ is the edge measure. Equations (3-6) can be easily extended to 3D.

\section{RESULTS}

We present some preliminary results of the proposed biquaternion based DT image processing approach on synthetic and real DT data. The real DT data consisted of $12 \mathrm{im}-$ ages of normal adult brains taken from the John Hopkins LBAM database [9]. The Fourier transform axis was set to $\mu=i+(1+I) j+(1-I) k$. The quaternion toolbox [10] was used for the MATLAB implementations of the Fourier transform and convolution.

Figure 1 shows the magnitude spectra of various synthetic images. Figures 1(a), 1(c), and 1(b) show tensors varying in shape and orientation along different directions, which is also reflected in their magnitude spectra. Figures 1(d) and 1(e) show tensors varying at low and high frequencies along the horizontal. We see that their magnitude spectra have high response in the expected low frequency and high frequency regions, respectively. Figure 1(f) shows a square synthetic image and its frequency response, which is a sync function. These results confirm the basic working of the Fourier transform on DTs. 


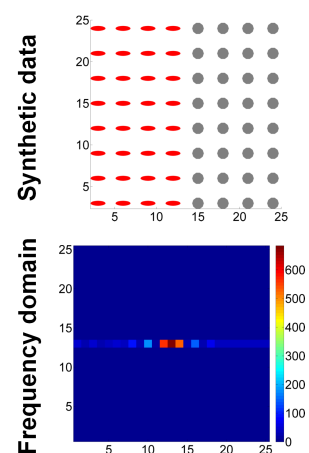

(a) Shape edge

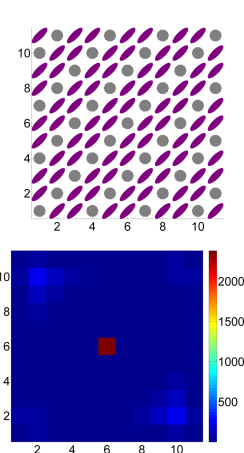

(b) Diag. variation

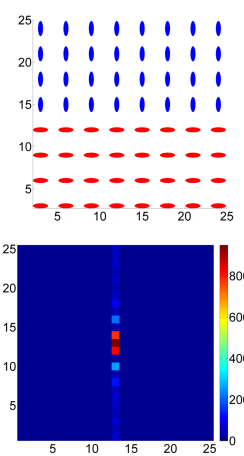

(c) Orientation edge
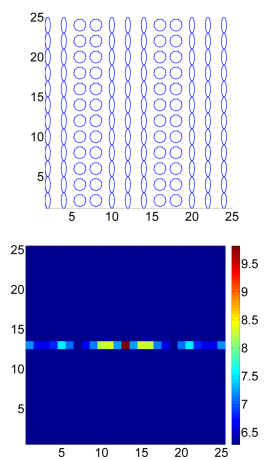

(d) Low freq.
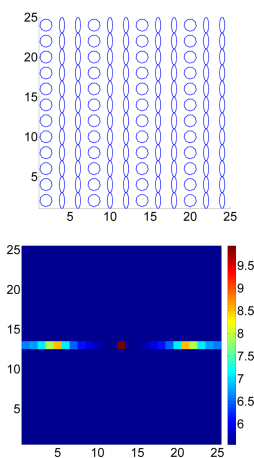

(e) High freq.
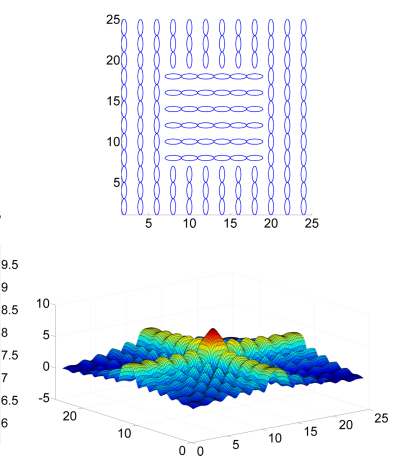

(f) Square image

Fig. 1. Synthetic examples (top row) and their magnitude spectrum (bottom row). (a) and (b): DTs varying in shape along the horizontal and diagonal. (c): DTs varying in orientation along the vertical. (d) and (e): DTs varying with different frequencies along the horizontal. (f) DTs oriented horizontally inside the square and vertically elsewhere.

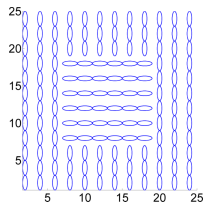

(a) Square image

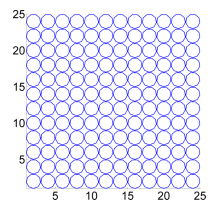

(b) All-stop

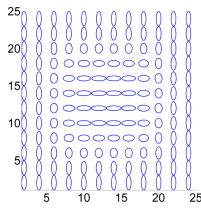

(c) Low-pass

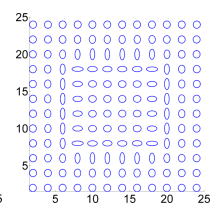

(d) High-pass

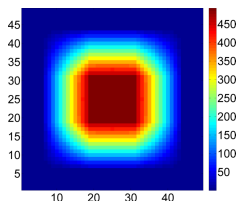

(e) Convolution

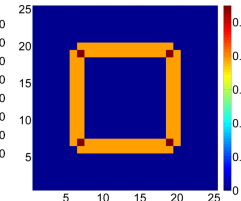

(f) Edge

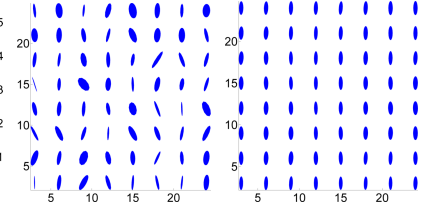

(g) Noisy

(h) Denoised

Fig. 2. Frequency filtering, convolution and edge detection on a square synthetic image (a). (b), (c) and (d) show all-stop, low-pass and high-pass frequency filtered images, respectively. (e) shows the biquaternion norm of the image convolved with itself. (f) shows edge strength using (6). (g) shows a noisy image of tensors oriented along the vertical and (h) shows the image after denoising.

Figure 2 shows frequency filtering, convolution and edge detection on the square synthetic image. Figure 2(b) shows the all-stop filtered image which has isotropic tensors everywhere $^{2}$. Figure 2(c) shows the low-pass filtered image, where the edges are blurred. Figure 2(d) shows the high-pass filtered image, where tensors are anisotropic along the edges and isotropic elsewhere. Figure 2(e) shows the biquaternion norm of the image convolved with itself (auto-convolution, or auto-correlation given the symmetric image), where we see a high response in the centre as expected. Figure 2(f) shows the proposed edge computed using (6). Figures 2(g), 2(h) show a noisy and denoised image after low pass filtering. These figures illustrate sample applications of the proposed tools and show that they work as expected.

Figure 3 shows compression of a DT image slice of a cross section of the Corpus Callosum (CC) obtained by truncating a fraction of the low energy Fourier coefficients. Note that even when $60 \%$ of the low energy Fourier coefficients are truncated, the energy lost is minimal and the shape and orientation characteristics of the DTs are largely retained.

Figure 4 illustrates the rotation property of the Fourier transform on a sagittal slice of the CC. Figure 4(c) is a $90^{\circ}$ rotated version of figure 4(a). The corresponding frequency

\footnotetext{
${ }^{2}$ Zero biquaternion decodes to a unit isotropic tensor in the DT domain.
}

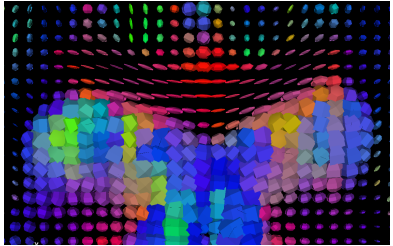

(a) $0 \%$ FT co-eff. truncated

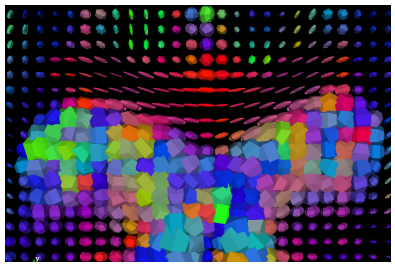

(c) $60 \%$ FT co-eff. truncated

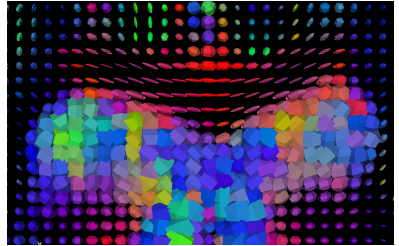

(b) $30 \%$ FT co-eff. truncated

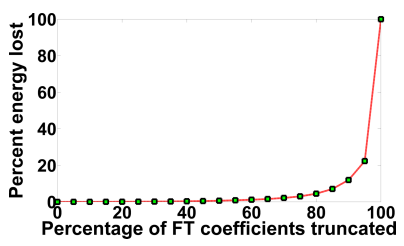

(d) Energy lost vs percent FT coeff. truncated
Fig. 3. DT image compression using Fourier transform. (a), (b) and (c) show reconstructed coronal slices of a cross section of the CC after a fraction of the low energy Fourier coefficients are truncated. (d) shows a plot of the energy lost for different levels of truncation. The energy lost was computed as the sum of squared magnitudes of the truncated coefficients. 


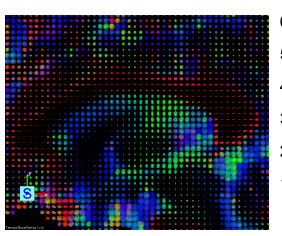

(a) Sagittal slice of CC

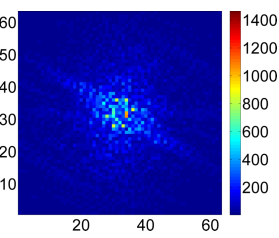

(b) Mag. spec.

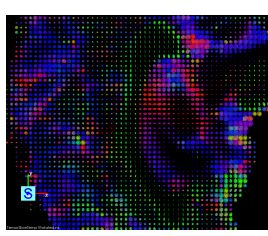

(c) Slice rotated by $90^{\circ}$

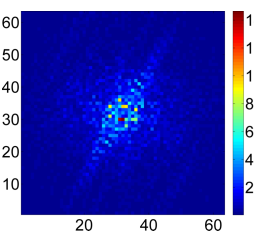

(d) Mag. spec.

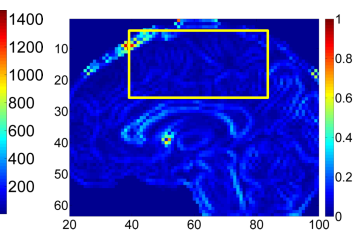

(e) Biquaternion edge

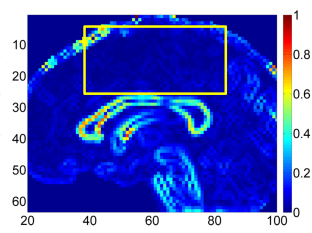

(f) FA edge

Fig. 4. Rotation of the magnitude spectrum and edge detection. (a) and (c) show a sagittal slice of the CC and its $90^{\circ}$-rotated version respectively. (b) and (d) show their corresponding magnitude spectra. (e) and (f) show edge detection using the proposed biquaternion gradient and FA gradient, respectively.

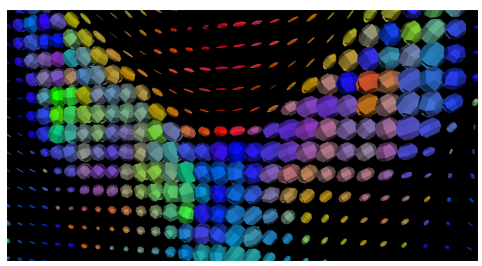

(a) DT image slice

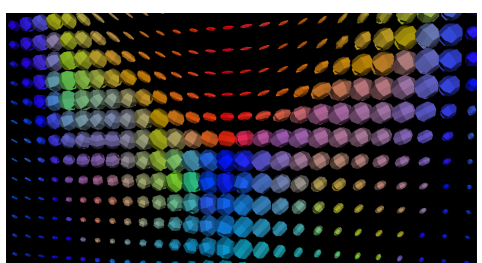

(b) Low pass filtering

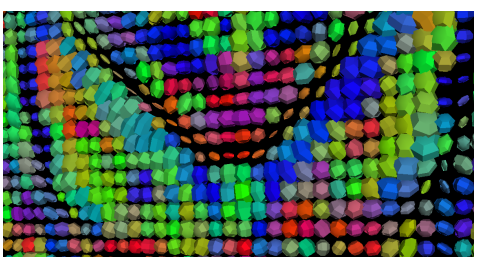

(c) High pass filtering

Fig. 5. Frequency filtering on real data. (a) lateral ventricle. (b) and (c): after low pass and high pass filtering, respectively.

spectrum is also rotated by $90^{\circ}$, as seen from figures 4 (b) and 4(d). Figure 4(e) shows the biquaternion based edge strength computed using (6). Edge computed from FA gradient is also shown for comparison in Figure 4(f). We see that the biquaternion based edge captures more structure than the FA based edge, demonstrating that the biquaternion gradient captures greater amount of information than the FA gradient.

Finally, figure 5 shows frequency domain filtering of a real DT slice containing the ventricle. We clearly see more regularized tensors in figure 5(b) and the highlighted edge in figure 5(c). These results show great potential for frequency domain processing of DT images.

\section{CONCLUSIONS}

We proposed a biquaternion formalism to model DT-MRI data. This approach enables us to process DT images in a holistic manner. Further, we introduced biquaternion based Fourier transform (3), convolution (4), gradient (5) and edge detection (6) for DT images. Preliminary results show great potential of our approach for various DT image processing applications including denoising, compression, interpolation, classification and registration.

\section{REFERENCES}

[1] C.-F. Westin, S.E. Maier, H. Mamata, A. Nabavi, F.A. Jolesz, and R. Kikinis, "Processing and visualization for diffusion tensor MRI," Medical Image Analysis, vol. 6, pp. 93-108, 2002.

[2] D. K. Jones, "Studying connections in the living human brain with diffusion MRI," Cortex, vol. 44, no. 8, pp. 936-952, 2008.

[3] G. Kindlmann, X. Tricoche, and C.-F. Westin, "Delineating white matter structure in diffusion tensor MRI with anisotropy creases," Medical Image Analysis, vol. 11, no. 5, pp. 492-502, 2007.

[4] L. O'Donnell, W. E. L. Grimson, and C.-F. Westin, "Interface detection in diffusion tensor MRI," in MICCAI, 2004, pp. 360-367.

[5] S.J. Sangwine, "Fourier transforms of colour images using quaternion, or hypercomplex, numbers," Electronics Letters, vol. 32, no. 21, pp. 1979 -1980, 1996.

[6] L. Shi, B. Funt, and G. Hamarneh, "Quaternion color curvature," in $I S \& T C I C, 2008$, pp. 338-341.

[7] V. Arsigny, P. Fillard, X. Pennec, and N. Ayache, "LogEuclidean metrics for fast and simple calculus on diffusion tensors," Magnetic Resonance in Medicine, vol. 56, no. 2, pp. 411-421, 2006.

[8] S. Said, N. Le Bihan, and S.J. Sangwine, "Fast complexified quaternion Fourier transform," IEEE Trans. on Signal Processing, vol. 56, no. 4, pp. 1522 -1531, 2008.

[9] S. Mori, "John Hopkins Medical Institute: Laboratory of Brain Anatomical MRI, in vivo human database," http://lbam.med.jhmi.edu/, accessed February 2009.

[10] S. J. Sangwine and N. Le Bihan, "Quaternion Toolbox for Matlab®," 2005, Software library available at: http://qtfm. sourceforge.net/. 